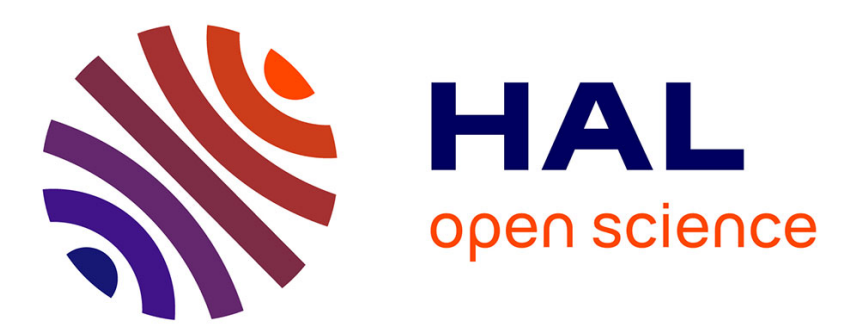

\title{
Répartition du potentiel électrocinétique imposée par un dipôle de courant excentré dans une plaque conductrice rectangulaire partiellement cloisonnée
}

\author{
J. Troquet, Ph. Lambin
}

\section{- To cite this version:}

J. Troquet, Ph. Lambin. Répartition du potentiel électrocinétique imposée par un dipôle de courant excentré dans une plaque conductrice rectangulaire partiellement cloisonnée. Revue de Physique Appliquée, 1985, 20 (6), pp.423-435. 10.1051/rphysap:01985002006042300 . jpa-00245355

HAL Id: jpa-00245355

https://hal.science/jpa-00245355

Submitted on 1 Jan 1985

HAL is a multi-disciplinary open access archive for the deposit and dissemination of scientific research documents, whether they are published or not. The documents may come from teaching and research institutions in France or abroad, or from public or private research centers.
L'archive ouverte pluridisciplinaire HAL, est destinée au dépôt et à la diffusion de documents scientifiques de niveau recherche, publiés ou non, émanant des établissements d'enseignement et de recherche français ou étrangers, des laboratoires publics ou privés. 


\title{
Répartition du potentiel électrocinétique imposée par un dipôle de courant excentré dans une plaque conductrice rectangulaire partiellement cloisonnée
}

\author{
J. Troquet et Ph. Lambin $(*)$ \\ Institut L. Fredericq de Physiologie, Université de Liège, 17, Place Delcour, 4020 Liège, Belgique \\ (*) Département de Physique des Facultés Universitaires N-D de la Paix, 61, rue de Bruxelles, 5000 Namur, Belgique
}

(Reçu le 27 juin 1983, révisé le 20 décembre 1984, accepté le 1er mars 1985)

\begin{abstract}
Résumé. - Connaissant les distributions de potentiel électrique qui apparaissent dans une plaque conductrice rectangulaire lorsque cette dernière est alimentée par une paire source-puits parallèle à l'un ou l'autre côté, nous comparons cette situation contrôle aux conséquences d'un cloisonnement de la plaque sur un quart de sa longueur par une ou deux fentes avec pour effet habituel de réduire la transmission de courant à l'entrée de la partie cloisonnée. Corrélativement, les cloisons introduisent des discontinuités dans la distribution de potentiel observée sur le côté auquel elles sont attachées. Selon le niveau de référence choisi, les informations cartographiques subissent des anamorphoses mais l'exploitation des données potentiométriques recueillies le long du contour complet de la plaque permet toujours de calculer les caractéristiques du dipôle équivalent au générateur connu et par conséquent, de vérifier l'exactitude du traitement. Dans ces conditions, négliger la partie cloisonnée ou la considérer comme non cloisonnée, conduit à des résultats faux; la possibilité de corrections cohérentes indique par ailleurs que les défauts de reconstruction du générateur ne sont pas imputables aux aléas expérimentaux. Transposées dans le domaine biologique, nos conclusions impliquent que limiter les sites de dérivation électrocardiographique au seul tronc en excluant les prolongements les plus proches du cour à savoir le cou et les bras, n'entraîne pas des erreurs moindres que celles que l'on veut éviter en définissant mieux la géométrie thoraco-abdominale.
\end{abstract}

\begin{abstract}
Knowing the potential distributions which arise on a conductive rectangular slab when the latter is energized by a source-sink pair parallel to one or the following side, we compare this control situation to the consequences of partitioning the slab over one quarter of its length by one or two splits and we observe that the flow transmission is partially impeded at the entry of the compartments separated by the splits. Correlatively, the partitions introduce discontinuities in the potential distribution along the side to which they are attached. According to the reference level, the mapped informations bear consistent changes but using the potentials measured along all the conductor boundaries, always enables to compute the characteristics of the dipole equivalent to the known generator and hence, to check the treatment. In such instances, neglecting the whole partitioned area or doing as if the partitions were absent, lead to false results, the correction of which rules out the possibility that the false results might come from experimental errors. Projected in the biological field, our conclusions imply that excluding the electrocardiographic information from the appendages to the trunk and near the heart namely the neck and the arms, lead to approximations not less than those we try to avoid by improving the definition for the thoraco-abdominal geometry.
\end{abstract}

\section{Introduction.}

Dans un précédent article (Lambin et Troquet 1983) [1] nous avons étudié la distribution de potentiel déterminée en tout point d'un cylindre conducteur sous l'effet de son alimentation par n'importe quel dipôle de courant.

La représentativité de ce modèle de l'action du cœur sur le corps humain est toutefois limitée principalement dans la direction de l'axe de révolution du cylindre. En effet, dans la direction céphalo-caudale, l'approximation de l'espace conducteur par des faces planes rend médiocrement compte d'une réalité dominée par les subdivisions scapulaire et pelvienne.

Nous traiterons ici ce nouveau problème ainsi que nous avions déjà abordé l'analyse du cylindre par 
celle de sa section $r, y$ soit un rectangle (Troquet et al., 1982) [2], pour cette fois, comprendre le phénomène de partition.

\section{Techniques.}

Dans ce but, utilisons à nouveau le réseau maillé dont nous nous sommes déjà servi. Rappelons que le réseau de résistances simule une plaque rectangulaire deux fois plus longue que large. Ici, nous subdivisons la plaque dans le sens de la longueur sur un quart de la distance disponible et à partir d'un seul côté. Pour simplifier la systématisation expérimentale, nous situons l'alimentation à mi-longueur c'est-à-dire à une demi-largeur de l'extrémité libre de la cloison et à deux demi-largeurs du côté non fendu. On peut ainsi espérer que le côté non fendu puisse ménager une référence assez pratique.

La disposition que nous adoptons rencontre en outre les faits biologiques à simuler puisque le cour se situe approximativement à égale distance du menton et des épines iliaques (Zero Potential Gradient Levels selon Nelson et al., 1971) [3] tandis que la subdivision scapulaire survient à peu près entre le cour et le menton. Fût-il réduit à deux dimensions, le simulacre de la partition scapulaire doit comprendre trois compartiments analogues au cou et aux deux bras. D'autre part, l'excentrement latéral du cour correspond approximativement au cloisonnement cou/bras gauche. Nous étudierons donc ces particularités. Il nous paraît néanmoins sage de commencer par des cas plus simples : soit un cloisonnement médian, évidemment plus représentatif de la subdivision pelvienne, avec une alimentation d'abord fixée au centre puis décalée latéralement par rapport au centre de la plaque.

Attendu que le cloisonnement s'étend sur un quart de la longueur, le niveau de l'alimentation est, dans la même direction, centré par rapport au raccord de la cloison et du côté tandis qu'il est excentré par rapport à l'extrémité libre de la subdivision.

En conséquence, nous estimons souhaitable de comparer la situation du réseau cloisonné non seulement à celle du réseau complet non subdivisé (alimentation centrée en $y$ ) mais encore à celle du réseau uniformément tronqué au niveau de l'extrémité libre de la subdivision (alimentation décentrée en $y$ ). Les résultats $\mathrm{du}$ cloisonnement s'inscrivent alors entre deux références limites déjà connues (Troquet et al., 1982) [2].

Les nouvelles distributions de potentiel sont construites à partir des mesures recueillies comme précédemment c'est-à-dire : alimentation Hewlett-Packard 6236A branchée sur une maille du réseau formé de 1640 résistances de $10 \mathrm{k} \Omega$ précises à $1 \%$ près et détection par voltmètre digital Philips PM 2421 par rapport au point milieu de l'alimentation.

Cette information redondante est le mieux appréciée sous forme de graphiques présentés en perspective cavalière où l'ordonnée correspond au potentiel local, l'abscisse repère la cote en longueur et l'étagement en profondeur est proportionnel à la position en largeur.

Pour montrer comment les données cartographiques varient avec le niveau de référence choisi alors que leur élaboration quantitative par la méthode de Gabor et Nelson, 1954 [4] échappe à cette contingence, nous utiliserons un exemple de calcul des caractéristiques du dipôle équivalent au générateur donné. A cette occasion, nous spécifierons quels rapports existent entre le potentiel à l'endroit du dipôle $\Phi_{0}$ et la moyenne $\Phi$ des potentiels de contour utilisés pour caractériser l'intensité et la position du dipôle équivalent.

Par la suite, nous ne traiterons plus que de ces

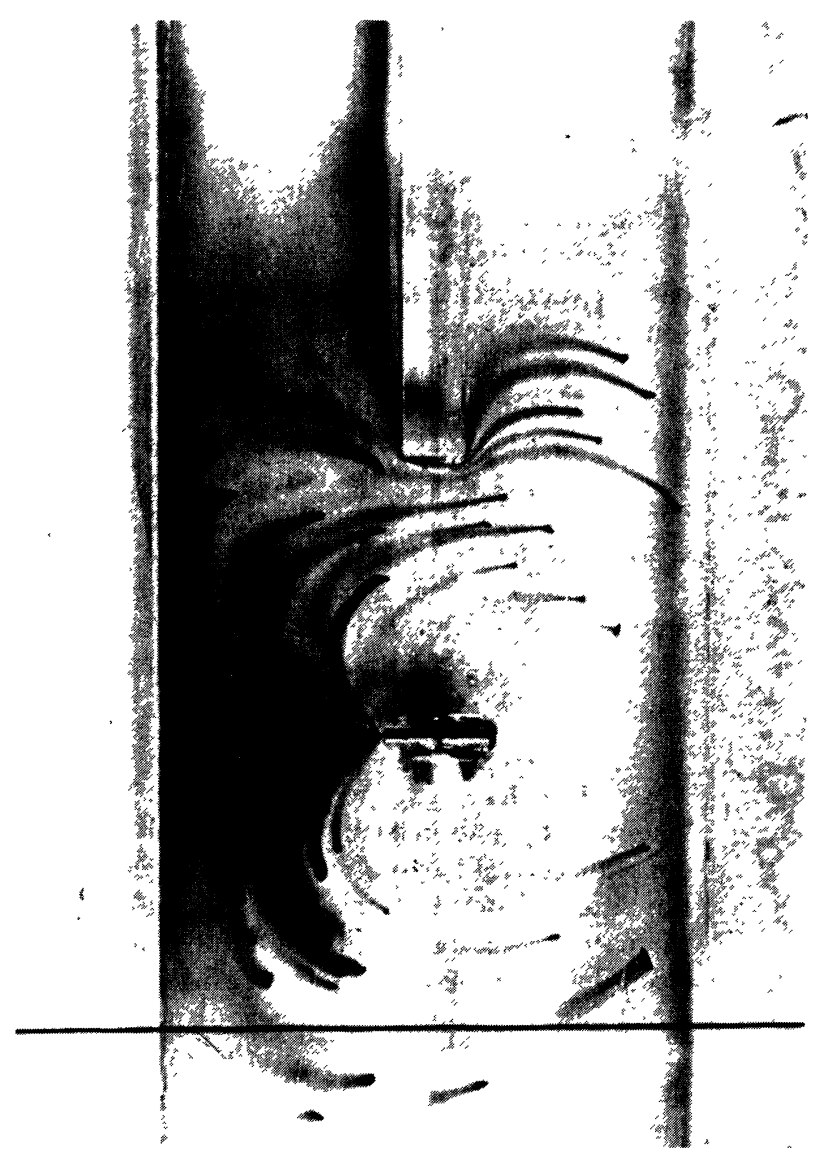

Fig. 1. - Au centre d'une cuve rectangulaire de faible profondeur et cloisonnée sur un quart de sa longueur, nous avons disposé une paire source-puits parallèle à la largeur. Nous observons les déplacements de fluide par leur marquage local au permanganate de potassium.

On remarque la pénétration des courants dans les compartiments situés de part et d'autre de la cloison.

[In the middle of a rectangular thin trough which is partitioned over a quarter of its length, we have a source-sink pair parallel to the width.

We observe the fluid lines traced by the potassium permanganate that they dissolve from crystals lying on the bottom. Notice how are washed the compartments on each side of the partition.] 
caractéristiques pour discuter les erreurs commises en négligeant les cloisons ou en substituant au contour complet un périmètre abrégé qui exclut la zone cloisonnée.

Dans les annexes mathématiques, nous justifierons enfin les principes retenus pour organiser nos observations en les fondant sur des considérations déductives.

Toutefois avant d'entamer ce programme, il n'est peut-être pas inutile de se rendre préalablement compte de ce que le cloisonnement ne supprime jamais complètement la circulation dans les compartiments qu'il limite latéralement et que, par conséquent, ceux-ci ne peuvent pas être considérés comme équipotentiels. A cet égard, recourons à une simulation hydraulique dans laquelle les filets de courant sont tracés par le permanganate de potassium qu'ils entraînent au fur et à mesure de sa dissolution à partir de cristaux répartis sur le fond du récipient dont les bords organisent l'écoulement forcé par des source et puits adossés.

\section{Résultats.}

Dans la figure 1, l'inévitable dilution du permanganate fait rapidement perdre la trace des filets de courant mais le raccord des segments lisibles indique très nettement le bossèlement des lignes par la cloison.
Un tel comportement s'oppose à la convexité uniforme du flux dans la moitié opposée du modèle où un trait repère le niveau comparable à celui de l'extrémité libre de la cloison.

On remarque également le parallélisme des filets de courant qui ont pénétré au-delà de l'extrémité libre de la cloison et l'alignement de leurs points de tangence à la direction transversale, sur des segments de droite parallèles à l'axe longitudinal. Dans le quadrant supérieur droit de la figure 2 , nous voyons les données potentiométriques recueillies dans des circonstances analogues sur le réseau de résistances. Par rapport à la situation illustrée dans le quadrant supérieur gauche, nous pouvons dire que la cloison introduit une discontinuité dans la distribution de potentiel observée le long du côté sur lequel la cloison est implantée. De plus, la discontinuité de cloison sépare des classes de valeurs beaucoup moins dispersées que dans les segments comparables des distributions continues.

Si par ailleurs, nous comparons les quadrants supérieur droit et inférieur gauche, nous constatons que la distribution de potentiel au niveau de l'extrémité libre de la cloison, indiquée en pointillé, reste continue et d'une amplitude inférieure à celle de la distribution au même niveau mais en l'absence de tout prolongement. Bien qu'elle ne soit pas soulignée par des pointillés la distribution correspondante
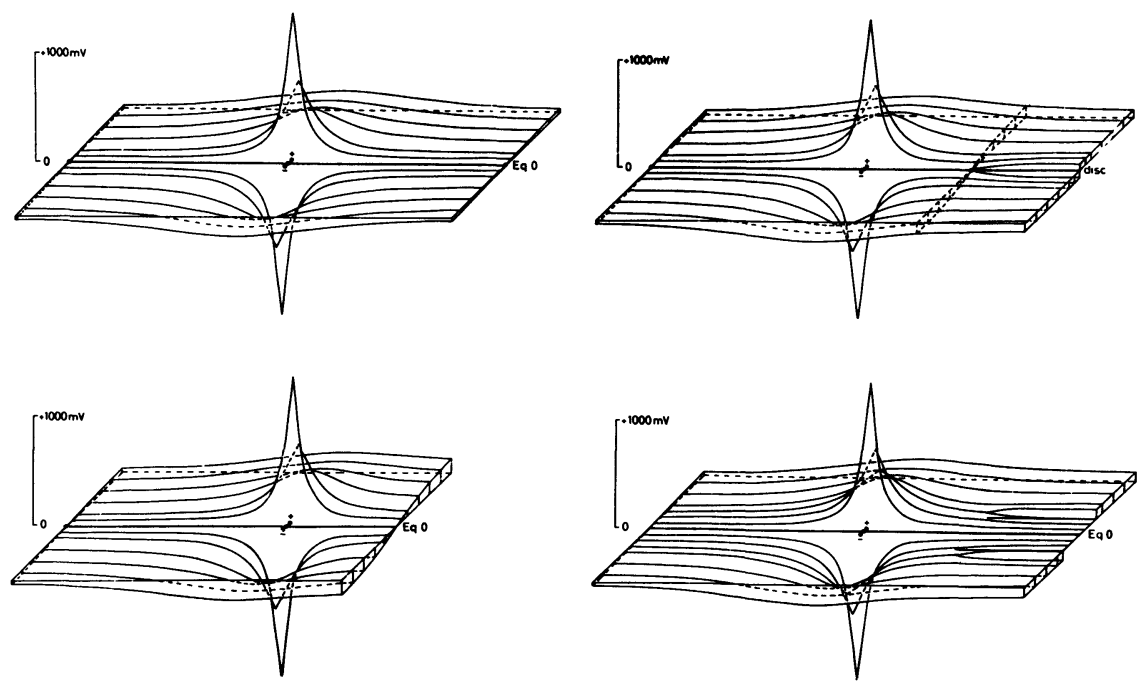

Fig. 2. - Distributions de potentiel $V$ dans le réseau alimenté par un générateur parallèle au petit côté et situé sur l'axe de symétrie longitudinale. Les perspectives cavalières suggèrent que le plan conducteur est vu de gauche et d'en haut et qu'il est recouvert ou creusé selon le signe des potentiels qu'il supporte en chaque endroit.

Dans l'ordre, les quarts supérieurs gauche et droit puis les quarts inférieurs gauche et droit correspondent à la structure contrôle, au réseau compartimenté par une cloison médiane, au conducteur tronqué enfin au système partiellement subdivisé en trois compartiments parallèles.

[Potential distributions in a network of resistors energized by a source sink pair parallel to the small side and situated on the longitudinal axis. The conductive plane is viewed from left and above to suggest that the potentials move the surface upwards or downwards according to their local sign.

Above and to the left, we have the control situation that is without any partitioning. On the same level but to the right, the network is axially partitioned.

Below and to the left, the last fourth of the network is suppressed while to the right, the same fourth is divided into three compartments. Notice the discontinuities in the potential distributions on the side bearing the partitions.] 
sur réseau non cloisonné (en haut et à gauche) est d'amplitude encore inférieure à celle que l'on observe à l'entrée du prolongement fendu (en haut et à droite). Il s'ensuit que l'obstacle représenté par cette dernière structure, s'inscrit entre un maximum évidemment lié à l'obturation des deux compartiments (réseau tronqué) et un minimum défini en l'absence de tout compartimentage du dernier quart du réseau. Enfin, si nous subdivisons le prolongement en trois compartiments (quadrant inférieur droit) l'obstacle dont témoigne l'amplitude de la distribution de potentiel au niveau de l'extrémité libre des deux cloisons s'accroît et se rapproche ainsi du maximum caractéristique d'un réseau tronqué au même endroit.

Dans les quatre cas, les distributions de potentiel sur le côté où sont éventuellement implantées les cloisons manifestent une parfaite symétrie par rapport à l'équipotentielle zéro ainsi qu'une moyenne nulle.

D'une manière générale, les équipotentielles et les lignes de courant fournissent des renseignements semblables. Ainsi, les intersections de l'équipotentielle nulle avec la paroi ou l'extrémité libre d'une cloison impliquent que le courant y est localement tangent. On comprend dès lors pourquoi les cloisons forcent certaines équipotentielles non nulles à leur devenir parallèles. Dans ces conditions en effet, les boucles de courant présentent un bossèlement qui multiplie les points de tangence à une même direction et, dans chaque compartiment, le point de tangence à la largeur se reproduit sur des filets pratiquement parallèles, donc le long d'un axe longitudinal. Est-il nécessaire de rappeler qu'au niveau du contour luimême et quelle que soit son orientation, le courant suit forcément la paroi et lui est partout tangentiel ?
Nous savons déjà (Troquet et al., 1982) [2] que l'excentrement retenu pour le générateur transversal $(0,3 \mathrm{demi}-$ largeur) amène l'équipotentielle nulle à émerger sur la longueur la plus proche du générateur de telle sorte que les deux largeurs manifestent des potentiels tous de même signe et en moyenne équivalents (en haut et à gauche dans la figure 3).

Pour sa part, le cloisonnement médian (en haut et à droite) dévie l'équipotentielle nulle vers la cloison avec à nouveau, la mise en place d'une distribution à deux classes qui, cette fois, ne sont plus symétriques par rapport à l'axe longitudinal. Ce comportement imposé à l'équipotentielle nulle apparente le cloisonnement à l'amputation du réseau (en bas et à gauche); cette dernière intervention ne parvient toutefois pas à déplacer l'équipotentielle nulle jusqu'au milieu de la largeur.

$\mathrm{Si}$ nous dédoublons la cloison, l'équipotentielle nulle rejoint par contre la cloison la plus proche du générateur. En multipliant les compartiments, l'équipotentielle nulle tendrait d'ailleurs vers l'intersection caractéristique du réseau tronqué qui à ce point de vue aussi, se présente comme une limite pour le processus de partition.

Quand l'alimentation de la plaque est longitudinale et centrée (Fig. 4), nous constatons que le cloisonnement médian reste sans effet sur la distribution de potentiel en largeur, en particulier au niveau de l'extrémité libre de la cloison ainsi qu'à hauteur de son implantation périphérique (quadrants supérieurs gauche et droit). Cette exception ne subsiste toutefois pas dès que nous dédoublons la cloison puisque nous observons alors trois classes de potentiel séparées par deux discontinuités. Est-il nécessaire de remarquer
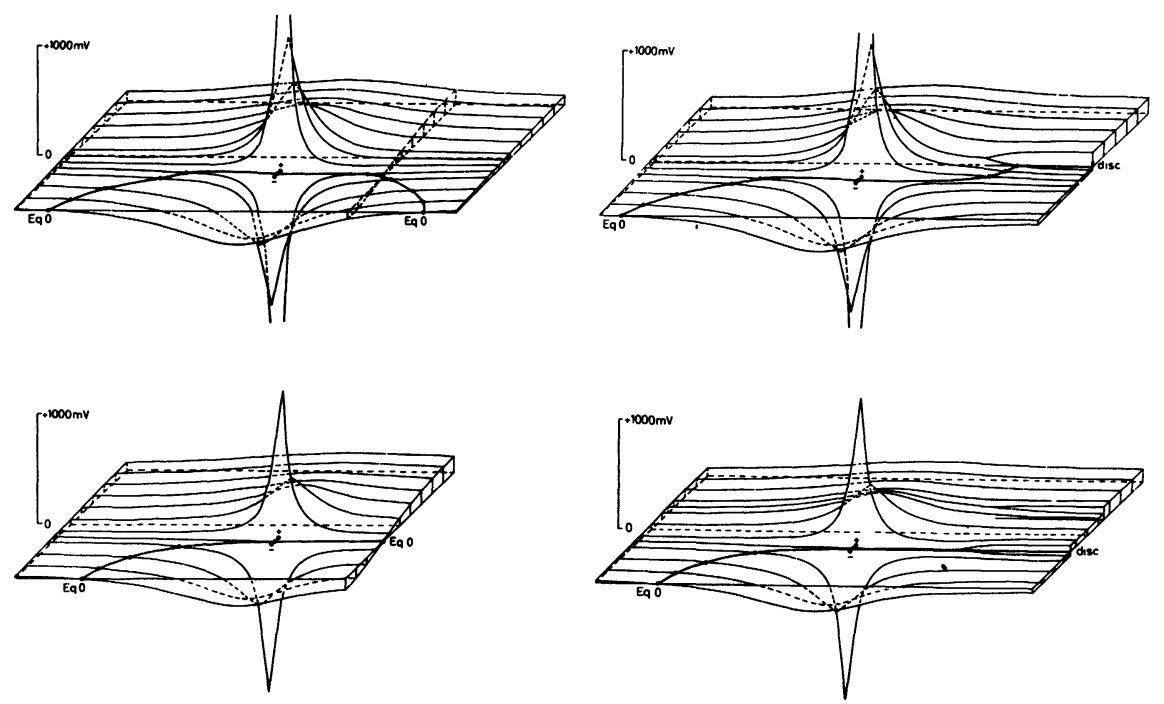

Fig. 3. - Même disposition que dans la figure 2 pour illustrer les conséquences potentiométriques de l'alimentation du réseau par un générateur transversal situé plus près d'un grand côté que de l'autre. Pour les commentaires, voir le texte.

[Same disposition as in figure 2 to illustrate the consequences of shifting the transversal dipole away from the longitudinal axis.

Notice how curved is the zero isopotential.] 

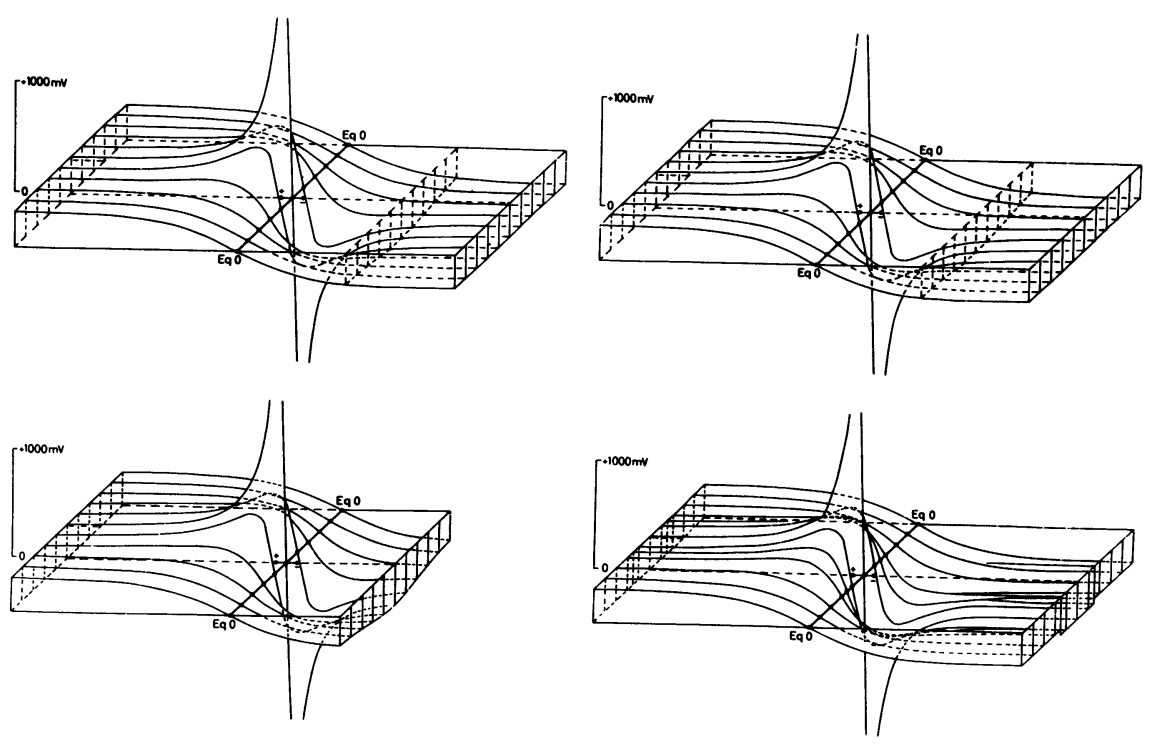

Fig. 4. - Présentation identique aux figures 2 et 3. Les résultats concernent cette fois une alimentation parallèle au grand côté et située sur l'axe de symétrie longitudinale du réseau. Pour les commentaires, voir le texte.

[Same disposition as in figures 2 and 3. The results are related to a dipole parallel to the length and situated on the longitudinal axis.

Notice that the axial partitioning is now of no consequence.]

que cette fois, la classe la plus ample est située sur l'axe de symétrie longitudinale où l'équipotentielle nulle a été remplacée par la ligne joignant les maxima de potentiel relevés à cote longitudinale constante ? De cette crête de potentiel se détachent évidemment les filets de courant qui épuisent progressivement le flux axial.

Avec une alimentation longitudinale et excentrée
(Fig. 5) nous retrouvons l'incurvation de cette crête de potentiel entre pôles et paroi ainsi que ses butées sur la longueur la plus proche du générateur (Troquet et al., 1982) [2]. Un cloisonnement médian a pour effet de ramener la butée correspondante à l'entrée du compartiment homolatéral (en haut et à droite) : situation à peine modifiée par le dédoublement de cloison (en bas et à droite).
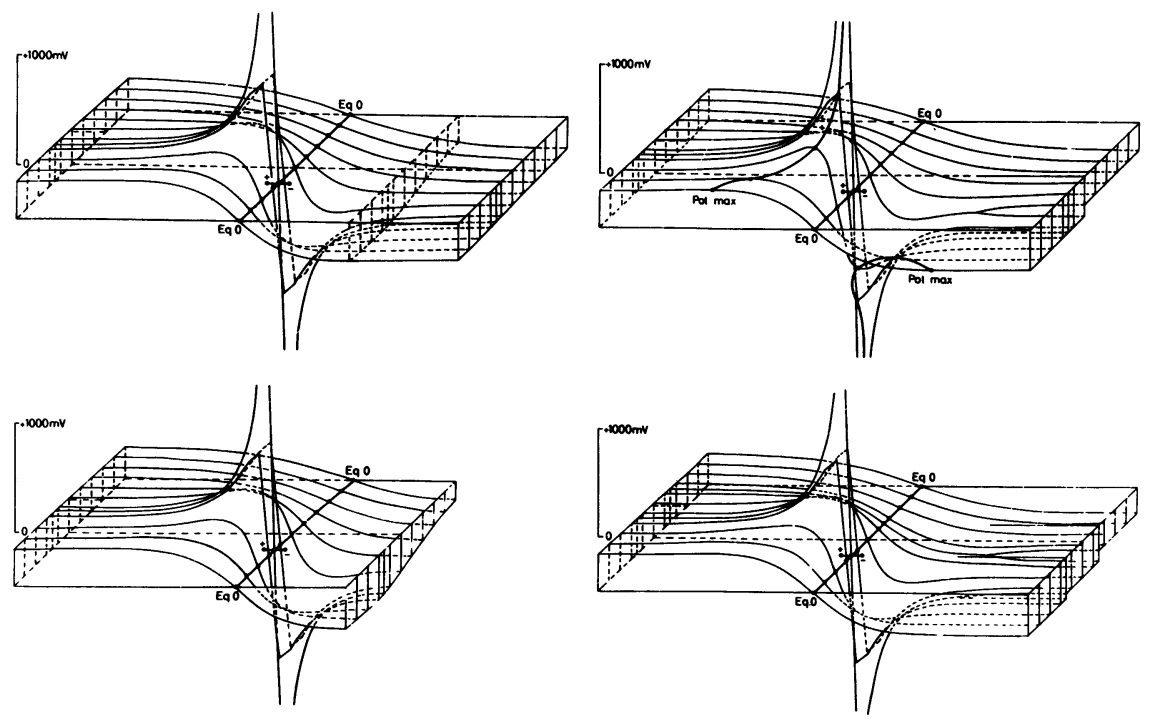

Fig. 5. - Les résultàts qui se rapportent à une alimentation parallèle à l'axe de symétrie longitudinale mais située en dehors de celui-ci, sont groupés comme précédemment (Figs. 2, 3 et 4) pour visualiser la description qui les concerne dans le texte.

[The disposition is again as previously but, here, the longitudinal dipole is shifted towards the proximal length. Notice how much curved is the line which comes from the pole to the boundary by joining each potential maximum observed along all the transversal levels.] 
L'illustration suivante (Fig. 6) montre la distribution de potentiel le long du contour d'une plaque rectangulaire une fois et demie plus longue que large (réseau tronqué). Le générateur est longitudinal $\left(M_{x}=0\right)$ et décentré $(X \neq 0)$. Attendu que l'origine des coordonnées est arbitrairement fixée à une demi-largeur des bords supérieur et latéraux, le dipôle est par construction, situé en $Y=0$ et en $X=+3$ éléments de discrétisation de la distance $(\mathrm{d} l)$. Le signe du décentrement indique déjà que nous suivons la convention vectocardiographique pour l'axe $x$; nous faisons de même pour l'axe $y$ (+ vers le bas). Cette convention est donc partiellement opposée à celle adoptée pour la représentation potentiométrique $(+$ à droite et en haut du contour pris pour ligne de base). Enfin, selon l'orientation relative de l'axe considéré et de la normale à la paroi, le potentiel conserve son signe $\left(0^{\circ}\right)$, s'annule $\left(90^{\circ}\right)$ ou s'inverse $\left(180^{\circ}\right)$.

Les surfaces comprises entre le contour et le profil potentiométrique représentent les composantes du moment de premier ordre de la distribution de poten- tiel qui caractérise le moment dipolaire en unités : $\mathrm{mV}$.d $l$ dont le produit par deux constantes : l'épaisseur $(h)$ et la conductivité $(\gamma)$ définit le générateur comme il se doit en mA.dl. En l'occurrence, nous symbolisons le calcul de la composante du moment dipolaire dans la direction $y$ soit $M_{y}$ par $\gamma \cdot h \int V \mathrm{~d} x$ En effet, l'intégrale du potentiel le long du contour $\oint V \mathrm{~d} l$ se réduit à l'intégrale de $V$ le long des deux largeurs : $\int V \mathrm{~d} x$; la contribution des deux longueurs étant par principe nulle. La valeur résiduelle de $\int V \mathrm{~d} y$ représente donc un $M_{x}$ dont le caractère négligeable sied à sa nature d'artefact. Sa présence rappelle néanmoins que la somme vectorielle $M$, de $M_{x}$ et de $M_{y}$ interdit que $M$ puisse être négatif. Les composantes des moments du second ordre

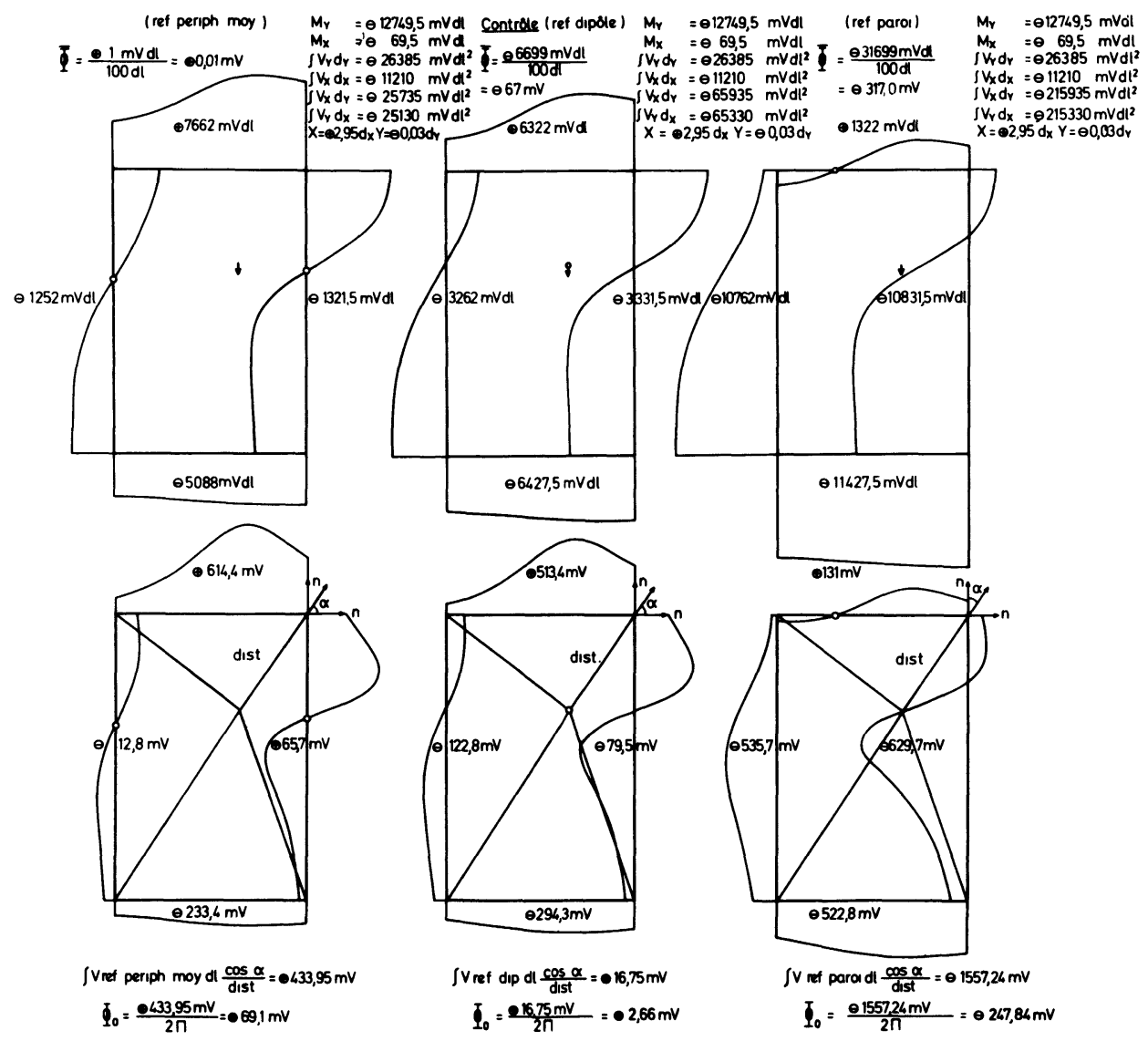

Fig. 6. - En haut, anamorphose d'une même distribution de potentiel sous l'effet d'un changement du niveau de référence. En bas, les distributions correspondantes après une pondération telle qu'on puisse en déduire le potentiel au centre du dipôle $\Phi_{0}$.

Pour les commentaires, voir le texte.

[Above, we see the potential distribution generated by an eccentric dipole along the perimeter taken as a base line. In the middle, the reference is situated on the dipole mid-point. On the left, the reference is shifted just to nullify the potential mean on the perimeter $\Phi$. On the right, the shift is arbitrary but in the three cases, the dipole characteristics are unaffected. Below, we see the corresponding distributions after weighting the potentials to deduce the mid-point level $\Phi_{0}$.] 
de la distribution sont également détaillées dans la figure 6 : ils servent à résoudre un système de deux équations à deux inconnues ( $X$ et $Y$ ) aboutissant au calcul de la position du générateur par rapport à l'origine des coordonnées

$$
\begin{aligned}
& X M_{x}-Y M_{y}=\int x V \mathrm{~d} y-\int y V \mathrm{~d} x \\
& X M_{y}+Y M_{x}=\int x V \mathrm{~d} x+\int y V \mathrm{~d} y
\end{aligned}
$$

En haut et au milieu, la distribution de potentiel est considérée par rapport au point milieu du dipôle. Nous renvoyons le lecteur au quadrant inférieur gauche de la figure 5 pour plus d'informations cartographiques. Celle que nous traitons ici par la méthode de Gabor et Nelson, 1954 [4] fournit une excellente caractérisation du dipôle équivalent au générateur connu.

Dans ce cas, le potentiel périphérique moyen $\Phi$ s'élève à plusieurs dizaines de $\mathrm{mV}$. Attendu que nous connaissons la position du dipôle, nous pouvons déduire (Annexe 1) un facteur de pondération qui tienne compte de la distance entre chaque point de la paroi et le centre du dipôle ainsi que de l'inclinaison de cette liaison par rapport à la normale pariétale aux mêmes points de mesure. L'intégration pondérée est observable sous forme graphique au milieu et en bas avec pour solde un résidu qui ne diffère pas significativement du potentiel au niveau du centre du dipôle $\Phi_{0}$.

Dans la partie gauche de la figure 6 , nous avons annulé $\Phi$ en retirant de chaque potentiel mesuré par rapport au centre du dipôle, la valeur périphérique moyenne obtenue dans les mêmes conditions. Il s'ensuit que la distribution est légèrement anamorphosée mais sans incidence sur les caractéristiques attribuées au dipôle équivalent au générateur inchangé. $\Phi_{0}$ est par contre décalé comme l'était antérieurement $\Phi$.

Dans la partie droite de la figure 6, nous avons enfin procédé à un décalage caricatural avec la même stabilité des résultats de la méthode de Gabor et Nelson, 1954 [4] aussi bien que de la différence $\Phi-\Phi_{0}$. Ayant ainsi vérifié que la méthode de Gabor et Nelson, 1954 [4] donne accès à des conclusions toujours univoques, nous l'avons appliquée aux distributions de potentiel recueillies dans des circonstances inspirées des contraintes biologiques à savoir celles d'un générateur décentré dans un réseau doublement cloisonné.

Le tableau I rassemble comme prévu, non seulement les valeurs correctement traitées en tenant compte des cloisons mais encore les approximations qui découlent soit de l'ignorance pure et simple de ces cloisons soit de l'exclusion globale de la partie cloisonnée du réseau.

Par rapport à la caractérisation contrôle, la sup-

REVUE DE PHYSIQUE APPLIQUÉE. - T. 20, № 6, JUIN 1985

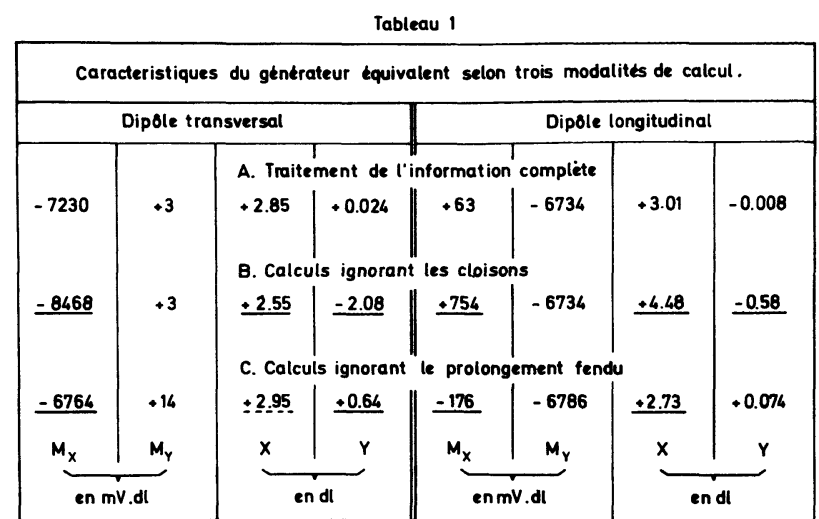

pression des composantes de cloison surestime de $17,5 \%$ l'intensité des dipôles transversaux mais reste sans effet sur le module des dipôles longitudinaux. Dans ce type d'alimentation, il apparaît en outre, une composante transversale fictive $\left(M_{x}=0,11 M_{y}\right)$.

La même simplification abusive du contour entraîne une légère sous-estimation de l'excentrement des générateurs transversaux $(11 \%)$ mais une surestimation importante de la position en $x$ des générateurs longitudinaux $(50 \%)$.

Inversement, la position en $y$ des dipôles longitudinaux se rapproche légèrement des cloisons tandis que le déplacement fictif des dipôles transversaux dans la même direction y est beaucoup plus accentuée. Attendu que la position contrôle en $y$ diffère très peu de zéro, l'importance relative de l'écart apparent deviendrait énorme si on devait choisir de le rapporter à l'écart contrôle. Nous minimisons par contre la réalité en appréciant l'écart par rapport au déplacement maximum dans la même direction longitudinale soit une largeur et en chiffrant ainsi les erreurs susmentionnées à 2,5 et $12 \%$.

Dans la pratique, il est plus habituel de ne pas tenir compte du prolongement cloisonné ; on commet alors d'autres erreurs qui consistent en : 1) une sousestimation de l'intensité des dipôles transversaux de $6,5 \%$ toujours sans modification significative de l'intensité des dipôles longitudinaux mais avec l'apparition corollaire d'une composante transversale fictive $\left.\left(M_{x}=0,025 M_{y}\right) ; 2\right)$ une surestimation modérée de l'excentrement des dipôles transversaux (4\%); par ailleurs, une sous-estimation plus importante du même décentrement pour les dipôles longitudinaux $(11 \%)$; 3) enfin, une augmentation modérée (4\%) de la distance estimée entre la position en $y$ des dipôles transversaux et celle des extrémités libres des cloisons sans retentissement appréciable sur l'origine des dipôles longitudinaux.

Nous avons également analysé toutes les autres distributions en particulier, celles qui sont déterminées par des paires source-puits situées sur l'axe de symétrie longitudinale du réseau doublement cloisonné. Qu'il nous suffise de mentionner que les résultats sont les mêmes à part les erreurs de position en $x$ et la composante fictive $M_{x}$ que la symétrie fait disparaître. 
Nous avons même complété le modèle du tronc " bras levés » par un simulacre de jambes sans qu'une adjonction aussi distale modifie le tableau que nous venons de brosser.

\section{Discussion.}

Nous avons observé le phénomène de partition d'un conducteur en présence d'un excentrement du générateur qui l'alimente, selon les deux points de vue de l'électrocardiologie : celui des cartes de potentiel et celui de leur synthèse vectocardiographique. Comme nous l'avons vu, le niveau de référence de la distribution de potentiel n'a aucun retentissement sur le calcul des caractéristiques du dipôle équivalent; de même, les cartes hydrauliques correspondent toujours au gradient des cartes de potentiel ce qui retire toute importance aux valeurs absolues. En particulier, l'équipotentielle nulle doit être considérée comme le lieu géométrique des points dont le potentiel ne diffère pas de celui de la référence. Compte tenu de la symétrie de la paire source-puits, $i$ est certes commode mais non nécessaire que l'équipotentielle nulle passe par le centre du dipôle.

Si nous choisissons pour référence, la moyenne des potentiels périphériques, l'équipotentielle nulle ne passe par le centre du dipôle que dans le cas où la symétrie du générateur se superpose à une symétrie semblable du milieu que le dipôle alimente soit le cas restrictif où l'axe perpendiculaire à celui du dipôle sépare le milieu homogène en deux moitiés égales. En dehors de cette condition, la différence entre $\Phi$ et $\Phi_{0}$ s'accroît d'autant plus qu'augmente l'excentrement.

Pour connaître la constante à ajouter à chaque potentiel périphérique afin que la distribution ainsi corrigée présente une équipotentielle nulle qui passe par le centre du dipôle, il faut pondérer les potentiels originaux par la distance entre chaque point de mesure et le centre du dipôle ainsi que par l'orientation de cette liaison par rapport à la normale pariétale aux mêmes endroits. Mais cette manipulation requiert la connaissance préalable de la position du dipôle, c'est-à-dire l'application des équations de Gabor et Nelson 1954 [4]. Ce n'est donc pas un mince paradoxe pour ceux qui refusent d'intégrer les détails de leurs cartes de potentiel de devoir néanmoins recourir au calcul de la position d'un générateur équivalent pour présenter leur information selon la référence qui lui convient le mieux.

A vrai dire, la référence réellement utilisée in vivo est le plus souvent le central terminal de Wilson c'est-à-dire la moyenne des potentiels des bras gauche et droit ainsi que de la jambe gauche qui, dans l'hypothèse d'un cour au centre d'un espace homogène ne peut pas différer du potentiel au point milieu du dipôle équivalent au myocarde. (Taccardi 1963) [5].

Le caractère idéal du central terminal de Wilson est dénoncé de longue date (Frank 1953) [6] mais peu de perfectionnements constructifs ont été proposés. Le plus intéressant d'entre eux est dû à Bayley et al. 1954 [7] au départ du fait suivant : quel que soit l'excentrement dipolaire, l'équipotentielle nulle occupe tout le contour périphérique lorsque ce dernier est représenté par un shunt c'est-à-dire lorsque la conductivité externe devient infinie. Cette condition limite et opposée à celle qui prévaut normalement dans l'air, remanie évidemment les distributions de potentiel et de courant. L'étude de ces changements en fonction des conductivités interne et externe a pour auteurs Nelson et Gastongay 1959 [8]. En les citant trop brièvement, nous ne voudrions pas minimiser les travaux des chercheurs qui ont abordé le sujet. Nous nous sentons toutefois proches de Bürger 1955 [9] et de Plonsey 1963 [10] qui insistent sur la vanité de vouloir définir le phénomène électrocardiographique dans un cadre absolu. Au-delà de ce préalable, notre description des effets de cloisonnement les inscrit entre deux niveaux déjà connus par limitation de l'espace accessible au courant produit par le générateur. En moyenne, tout se passe comme si les cloisons agissaient en rapprochant l'extrémité correspondante du réseau. Cette équivalence ne doit toutefois pas faire oublier que l'effet n'est pas identifiable à celui d'une interface entre milieux de résistivité différente (Troquet et al. 1985) [11] mais est obtenu par des changements autrement localisés des conditions aux limites. Nous n'en voulons pour illustration que l'absence d'effet d'une cloison située dans l'axe d'un dipôle longitudinal centré. Ainsi, la symétrie implique déjà que $V=f(x)$ culmine sur l'axe avec pour conséquence que $\frac{\partial V}{\partial x}=0$ : condition que la cloison ne peut que répéter sans introduire quoi que ce soit de neuf. Plus simplement, aucun courant ne traverse alors la ligne médiane fût-ce en l'absence de cloison.

En fait, le supplément de conditions aux limites introduit par les cloisons est le mieux traduit par les discontinuités à ces cloisons. Cette observation privilégiée ne doit cependant pas occulter la continuité potentiométrique le long d'un contour auquel les cloisons ajoutent des détours mais pas d'interruption. Quand nous comparons la distribution de potentiel à l'entrée des compartiments délimités par les cloisons à celle qui résulte de la suppression, au même niveau, de tout prolongement conducteur, la moyenne des mesures peut rester nulle dans chaque cas, par exemple en alimentation transversale centrée, ce qui imposerait une comparaison des variances. Ce point de vue descriptif risque.cependant de faire oublier que la comparaison est en principe illégitime dans la mesure où dans un cas la frontière est isolante tandis que dans l'autre elle est perméable au courant. La restriction précédente est de toute manière impérieuse en ce qui concerne le calcul des caractéristiques du dipôle équivalent au moyen des équations de Gabor et Nelson 1954 [4] telles que nous les avons rappelées plus haut. C'est d'ailleurs pourquoi nous dénonçons comme approximative une telle application et avec elle, la limitation des sites de dérivation électrocardiogra- 
phique au seul tronc. Pour apprécier l'erreur commise sans changer de formulation, il suffit de ne rien négliger du contour réellement isolant.

Il est toutefois possible de mieux comprendre l'erreur et par conséquent de la corriger en recourrant à une forme plus générale des équations de Gabor et Nelson 1954 [4] que nous avons utilisée pour traiter une interface entre milieux de conductivité différente c'est-à-dire un autre type de frontière perméable au courant (Troquet et al. 1985) [11]. Il n'entre néanmoins pas dans nos vues de démontrer d'une autre manière ce qui est déjà prouvé ici. Au plus voulons-nous indiquer pourquoi $M_{y}$ reste inchangé même si nous excluons le prolongement cloisonné du réseau qu'il alimente. Dans ce cas en effet, la formule générale :

$$
M_{y}=\frac{1}{\gamma}\left(\int V \mathrm{~d} x+\int E_{y} y \mathrm{~d} x+\int E_{x} y \mathrm{~d} y\right)
$$

se réduit à :

$$
M_{y}=\frac{1}{\gamma} \int V \mathrm{~d} x
$$

car la composante du champ électrique dans la direction $x, E_{x}$ est évidemment nulle le long des frontières longitudinales du conducteur tandis que l'intégration de la composante du champ électrique dans la direction $y, E_{y}$ s'avère nulle à l'entrée de chaque compartiment dans lesquels il pénètre autant de courant qu'il n'en sort et cela avec une densité inversement proportionnelle à l'écart entre le point de mesure et l'axe du dipôle.

\section{Remerciements.}

Nous remercions le Professeur R. Evrard pour son amical intérêt ainsi que pour ses remarques toujours judicieuses.

\section{Annexes.}

1) Relation entre $\Phi_{0}$ et le potentiel périphérique.

Considérons le générateur au centre $\mathbf{r}^{\prime} \mathrm{d}^{\prime} u n$ cercle de rayon $\varepsilon$ au sein du conducteur restant. Dans la couronne extérieure, nous pouvons écrire :

$$
\iint \Phi(\mathbf{r}) \Delta \ln \frac{1}{\left|\mathbf{r}-\mathbf{r}^{\prime}\right|} \mathrm{d}^{2} r=0
$$

puisque $\Delta \ln \frac{1}{\left|\mathbf{r}-\mathbf{r}^{\prime}\right|}$ est nul dans cette couronne. En outre, attendu que la même couronne est passive : $\Delta \Phi(\mathbf{r})=0$; que son contour extérieur est isolant $: \nabla \Phi(\mathbf{r}) \cdot \mathbf{n}_{\mathbf{e}}=0$ et que $\nabla \ln \frac{1}{\left|\mathbf{r}-\mathbf{r}^{\prime}\right|}=-\frac{\left(\mathbf{r}-\mathbf{r}^{\prime}\right)}{\left|\mathbf{r}-\mathbf{r}^{\prime}\right|^{2}}$, l'application de la formule de Green conduit finalement à :

$$
\oint_{\mathrm{e}} \Phi(\mathbf{r}) \frac{\left(\mathbf{r}-\mathbf{r}^{\prime}\right)}{\left|\mathbf{r}-\mathbf{r}^{\prime}\right|^{2}} \cdot \mathbf{n}_{\mathrm{e}} \mathrm{d} l=\oint_{\mathbf{i}} \Phi(\mathbf{r}) \frac{\left(\mathbf{r}-\mathbf{r}^{\prime}\right)}{\left|\mathbf{r}-\mathbf{r}^{\prime}\right|^{2}} \cdot \mathbf{n}_{\mathrm{e}} \mathrm{d} l+\oint_{\mathrm{i}} \ln \frac{1}{\left|\mathbf{r}-\mathbf{r}^{\prime}\right|} \nabla \Phi(\mathbf{r}) \cdot \mathbf{n}_{\mathrm{e}} \mathrm{d} l .
$$

Dans le second membre de cette équation $\mathbf{n}_{e}$ désigne la normale extérieure à la circonférence de rayon $\varepsilon\left(\mathbf{n}_{\mathbf{e}}=-\mathbf{n}_{\mathbf{i}}\right)$ le long de laquelle on intègre avec pour résultat :

$$
\oint_{\mathrm{e}} \Phi(\mathbf{r}) \frac{\left(\mathbf{r}-\mathbf{r}^{\prime}\right)}{\left|\mathbf{r}-\mathbf{r}^{\prime}\right|^{2}} \cdot \mathbf{n}_{\mathrm{e}} \mathrm{d} l=\frac{1}{\varepsilon} \oint_{\mathbf{i}} \Phi(\mathbf{r}) \mathrm{d} l+\ln \frac{1}{\varepsilon} \oint_{\mathbf{i}} \nabla \Phi(\mathbf{r}) \cdot \mathbf{n}_{\mathrm{e}} \mathrm{d} l .
$$

Par ailleurs, les éléments de source et de puits sont en même nombre, donc, en vertu du théorème de Gauss $\oint_{\mathrm{i}} \nabla \Phi(\mathbf{r}) \cdot \mathbf{n}_{\mathrm{e}} \mathrm{d} l=0$. D'autre part, $\Phi(\mathbf{r})$ pour un dipôle est égal à

$$
\Phi_{0}+\frac{\mathbf{p} \cdot\left(\mathbf{r}-\mathbf{r}^{\prime}\right)}{2 \pi \gamma\left|\mathbf{r}-\mathbf{r}^{\prime}\right|^{2}}+F\left(\mathbf{r}-\mathbf{r}^{\prime}\right)
$$

où, dans le second membre, le premier terme est une constante arbitraire, le deuxième représente le dipôle dans un plan infini et le troisième caractérise l'effet du contour e (potentiel image).

Rien ne s'oppose à ce que nous prenions lim. de $F\left(\mathbf{r}-\mathbf{r}^{\prime}\right)=0$ quand $\mathbf{r}$ tend vers $\mathbf{r}^{\prime}$; il s'ensuit que :

$$
\oint_{\mathrm{e}} \Phi(\mathbf{r}) \frac{\left(\mathbf{r}-\mathbf{r}^{\prime}\right)}{\left|\mathbf{r}-\mathbf{r}^{\prime}\right|^{2}}: \mathbf{n}_{\mathrm{e}} \mathrm{d} l=\frac{1}{\varepsilon} \oint_{0}^{2 \pi} \Phi_{0} \varepsilon \mathrm{d} \theta
$$


et par conséquent :

$$
2 \pi \Phi_{0}=\oint_{\mathrm{e}} \Phi(\mathbf{r}) \frac{\left(\mathbf{r}-\mathbf{r}^{\prime}\right)}{\left|\mathbf{r}-\mathbf{r}^{\prime}\right|^{2}} \cdot \mathbf{n}_{\mathrm{e}} \mathrm{d} l=\oint_{\mathrm{e}} \Phi \frac{\cos \alpha}{\text { dist. }} \mathrm{d} l
$$

$\Phi_{0}$ est ainsi défini comme la limite de $\Phi(\mathbf{r})$ pour $\mathbf{r}$ tendant vers $\mathbf{r}^{\prime}$, limite prise le long d'une trajectoire perpendiculaire au dipôle et passant par son centre, c'est-à-dire le potentiel au centre du dipôle.

2) Déduction de l'effet de cloisonnement dans une plaque infiniment longue A) Dipôle transversal (parallèle à l'axe $\mathrm{Ox}$ ). Considérons une plaque de largeur $2 a$ et qui s'étend de $-\infty$ à $+\infty$ dans la direction $O y$. Elle est cloisonnée le long de son axe de symétrie sur sa demi-longueur $O \leqslant y \leqslant+\infty$. En amont de la cloison, se trouve un dipôle de moment $p$ orienté parallèlement à l'axe $O x$ et placé au point de coordonnées $y^{\prime}=-y_{0}$ et $x^{\prime}=0$ $\left(y_{0} \geqslant 0\right)$.

On distingue deux régions : l'une où $x$ est compris entre + ou $-a$ tandis que $y \leqslant 0$; l'autre où $y \geqslant 0$ et où $x$ a pour limites 0 et + ou $-a$. Dans la première région, où se trouve le dipôle, le potentiel $\Phi_{1}(x, y)$, solution de l'équation inhomogène de Poisson résulte de la superposition de la solution générale de l'équation homogène de Laplace et d'une solution particulière de l'équation de Poisson. Ecrivons donc :

$$
\Phi_{1}(x, y)=V(x, y)+\psi(x, y)
$$

où $\psi$ symbolise la solution générale de l'équation de Laplace et où $V$ résulte du dipôle et de toutes ses images. Attendu que le potentiel dû à un dipôle placé au point $\left(x^{\prime}, y^{\prime}\right)$ est donné par :

$$
V=\frac{p\left(x-x^{\prime}\right)}{2 \pi \gamma\left[\left(y-y^{\prime}\right)^{2}+\left(x-x^{\prime}\right)^{2}\right]}
$$

$V$ peut aussi être exprimé par la différence des deux séries suivantes :

$$
\frac{p}{2 \pi \gamma}\left\{\sum_{k=-\infty}^{+\infty} \frac{x-4 k a}{\left(y+y_{0}\right)^{2}+(x-4 k a)^{2}}-\sum_{l=-\infty}^{+\infty} \frac{x-2 a+4 l a}{\left(y+y_{0}\right)^{2}+(x-2 a+4 l a)^{2}}\right\} .
$$

La première série représente la famille des images de même orientation que le dipôle original; la seconde est caractérisée par une orientation inverse. Quant au terme d'ordre nul, il indique respectivement le dipôle luimême $(k=0)$ et sa première image inversée $(l=0)$.

Après transformations adéquates en série de Fourier, on obtient :

$$
V(x, y)=\frac{p}{2 a \gamma} \sum_{n=1}^{\infty} \sin \left[(2 n+1) \frac{\pi x}{2 a}\right] \exp \left[-(2 n+1) \frac{\pi\left|y+y_{0}\right|}{2 a}\right] .
$$

Cette solution satisfait aux conditions aux limites imposées à $\Phi_{1}$ soit $\frac{\partial \Phi_{1}}{\partial x}=0$ pour $x= \pm a$ et la limite de $\Phi_{1}$ est finie pour $y$ tendant vers $-\infty$.

La solution générale de l'équation de Laplace qui vérifie les mêmes conditions aux limites est une superposition de fonctions harmoniques du type : $\varphi_{k}=\left(\alpha \mathrm{e}^{+k y}+\beta \mathrm{e}^{-k y}\right)(\gamma \cos k x+\delta \sin k x)$ où $\beta$ est nul puisque la solution doit être finie pour y tendant vers $-\infty$. D'autre part, si $\frac{\partial \Phi_{k}}{\partial x}=0$ en $x= \pm a, k=\frac{m \pi}{2 a}$ et $\gamma \sin \frac{m \pi}{2}=$ $\delta \cos \frac{m \pi}{2}$ avec pour conséquence une solution en cosinus si $m$ est pair et une solution en sinus si m est impair. Posant $m=2 n+1$, nous écrivons donc :

$\psi(x, y)=\frac{p}{2 a \gamma}\left\{\sum_{n=0}^{\infty} A_{n} \sin (2 n+1) \frac{\pi x}{2 a} \exp \left[+(2 n+1) \frac{\pi y}{2 a}\right]+C_{0}+\sum_{n=1}^{\infty} C_{n} \cos 2 n \pi \frac{x}{2 a} \exp \left(+2 n \pi \frac{y}{2 a}\right)\right\}$

dont nous ne retiendrons que la solution en sinus pour obtenir une fonction impaire en $x$ avec une constante de potentiel telle que $\Phi_{1}(x, y)=0$ pour $x=0$.

En ajoutant $\psi$ à $V$, on aboutit à :

$$
\Phi_{1}(x, y)=\frac{p}{2 a \gamma} \sum_{n=0}^{\infty}\left[A_{n} \exp \left[+(2 n+1) \frac{\pi y}{2 a}\right]+\exp \left[-(2 n+1) \frac{\pi\left|y+y_{0}\right|}{2 a}\right]\right] \sin \left[(2 n+1) \frac{\pi x}{2 a}\right] .
$$


Dans la région 2, le potentiel $\Phi_{2}(x, y)$ vérifie l'équation de Laplace et doit être tel que $\frac{\partial \Phi_{2}}{\partial x}=0$ en $x=0$ et $x=+a$ ou $-a$ et que la limite de $\Phi_{2}(x, y)$ soit finie pour y tendant vers $+\infty$. $\Phi_{2}$ est donc une superposition de fonctions harmoniques du type :

$$
\varphi_{k}(x, y)=(\alpha \cos k x+\beta \sin k x) \mathrm{e}^{-k y} \quad(k \geqslant 0)
$$

et par conséquent, selon que l'on considère la région $(0<x<+a)$ ou $(0>x>-a)$,

$$
\Phi_{2}= \pm \frac{p}{2 a \gamma}\left[B_{0}+\sum_{n=1}^{\infty} B_{n} \cos \frac{(n \pi x)}{a} \exp \left(-\frac{n \pi y}{a}\right)\right]
$$

En $y=0$, il doit y avoir continuité du potentiel et de sa dérivée par rapport à $y$ c'est-à-dire que d'une part :

$$
\sum_{n=0}^{\infty}\left[A_{n}+\exp \left[-(2 n+1) \frac{\pi y_{0}}{2 a}\right]\right] \sin \left[(2 n+1) \frac{\pi x}{2 a}\right]= \pm\left[B_{0}+\sum_{k=1}^{\infty} B_{k} \cos \frac{k \pi x}{a}\right]
$$

et que d'autre part,

$$
\sum_{n=0}^{\infty}(2 n+1)\left[A_{n}-\exp \left[-(2 n+1) \frac{\pi y_{0}}{2 a}\right]\right] \sin \left[(2 n+1) \frac{\pi x}{2 a}\right]= \pm\left[2 \sum_{k=1}^{\infty} k B_{k} \cos \frac{k \pi x}{a}\right] .
$$

En multipliant les deux membres de ces équations par $\sin \left[(2 l+1) \frac{\pi x}{2 a}\right]$ et en intégrant par rapport à $x$ entre $-a$ et $+a$, on tire de la première relation :

$$
A_{n}+\exp \left[-(2 l+1) \frac{\pi y_{0}}{2 a}\right]=\frac{4}{\pi} \sum_{k=0}^{\infty} \frac{2 l+1}{(2 l+1)^{2}-4 k^{2}} B_{k}
$$

et de la seconde :

$$
A_{n}-\exp \left[-(2 l+1) \frac{\pi y_{0}}{2 a}\right]=\frac{-8}{\pi} \sum_{k=1}^{\infty} \frac{k B_{k}}{(2 l+1)^{2}-4 k^{2}}
$$

Soustraire la dernière relation de la pénultième aboutit enfin à

$$
\sum_{k=0}^{\infty} \frac{B_{k}}{2 l+1-2 k}=\frac{\pi}{2} \exp \left[-(2 l+1) \frac{\pi y_{0}}{2 a}\right] \quad(l=0,1,2, \ldots, L, L \rightarrow \infty)
$$

ce qui permet de former un système de $L+1$ équations à $L+1$ inconnues. En fixant $y_{0} / a$, ces développements analytiques fournissent le moyen de calculer avec la précision que fixe le choix $L$ du nombre de termes des séries, les distributions de potentiel depuis leur valeur asymptotique en $y=+\infty$ jusqu'au moindre détail de leur conformation : qu'il s'agisse du profil potentiométrique le long du raccord entre les parties cloisonnée et non cloisonnée de la plaque ou de tout autre niveau parallèle à ce raccord. En se rapprochant du dipôle, on voit en particulier le maximum de cette distribution $\Phi=f(x)_{\bar{y}}$ quitter la paroi pour rejoindre progressivement la ligne médiane et l'atteindre au pôle comme il se doit. La figure 7 précise aussi l'établissement de la discontinuité de potentiel en suivant la cloison à partir de son extrémité centrale.

B) Dipôle longitudinal (parallèle à l'axe $\mathrm{Oy}$ ). Dans ce cas, une cloison médiane est sans effet, il nous faut donc traiter un double cloisonnement. A cette fin, nous définissons trois types de régions : 1) celle qui n'est pas compartimentée et contient le dipôle; 2) celles qui sont comprises entre les parois externes et les deux cloisons et dont les cotes en $x$ par rapport à la ligne médiane sont respectivement $\pm a$ et + ou $-c ; 3$ ) celle qui s'étend symétriquement autour de 0 jusqu'à + ou $-c$. En reprenant le raisonnement établi pour le dipôle transversal, mais en retenant cette fois, la fonction paire en $x$, nous obtenons pour $\Phi_{1}(x, y)$

$$
\frac{p}{2 a \gamma}\left[\frac{1}{2}+\sum_{n=1}^{\infty} \exp \left[\frac{-n \pi\left|y+y_{0}\right|}{a}\right] \cos \left(\frac{n \pi x}{a}\right) \operatorname{Sgn}\left(y+y_{0}\right)+\sum_{n=0}^{\infty} A_{n} \exp \left(\frac{n \pi y}{a}\right) \cos \left(\frac{n \pi x}{a}\right)\right]
$$

où $\operatorname{Sgn}\left(y+y_{0}\right)$ vaut 1 si $\left(y+y_{0}\right)>0$ et -1 si $\left(y+y_{0}\right)<0$. 


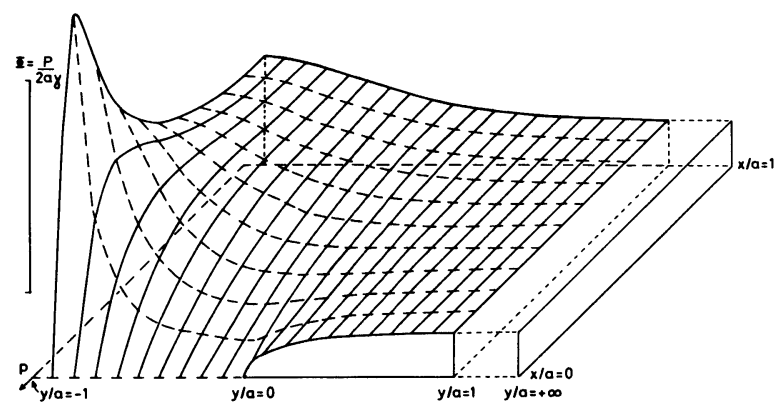

Fig. 7. - Distribution de potentiel $\Phi$ calculée en unités $p / 2 a \gamma$. Le dipôle transversal est situé à des coordonnées normalisées : $y / a=-1$ autrement dit à une demi-largeur de l'extrémité libre de la cloison et $x / a=1$ soit sur la ligne médiane. En $y / a=0$ débute la cloison et la discontinuité de $\Phi$. $\Phi(x)_{\bar{y}}$ manifeste des valeurs quasi asymptotiques en $y / a=1$; à l'opposé, la discontinuité de source implique une valeur infinie non représentable. La nappe couvre donc les variations les plus intéressantes de $\Phi$. On la superpose aisément au quart supérieur droit de la figure 2 construite à partir de données expérimentales.
[The potential distribution $\Phi$ has been simulated in $p / 2$ ay units. In normalized coordinates, the dipole lies on $y / a=-1$ and $x / a=0$. The partitioning and the associated discontinuity begin from $y / a=0$. Asymptotic values for $\Phi(x)$ are almost reached on $y / a=1$. So, our map shows the most significative $\Phi$ changes. They are easily compared with the corresponding ones in figure 2 drawn from experimental findings.]

Dans la région 3, l'absence de source allège à nouveau l'expression de $\Phi_{3}$ d'autant que les conditions aux limites ne sont pas plus nombreuses qu'en région 1 . Simplement, $\frac{\partial \Phi_{3}}{\partial x}=0$ est imposée en $x= \pm c$ au lieu de l'être en $x= \pm a$.

Il s'ensuit que $\Phi_{3}$ vaut :

$$
\frac{p}{2 a \gamma}\left[\sum_{n=0}^{\infty} C_{n} \exp \left(\frac{-n \pi y}{c}\right) \cos \left(\frac{n \pi x}{c}\right)\right]
$$

Dans la région 2, la source n'est pas plus présente; par contre, $\frac{\partial \Phi_{2}}{\partial x}=0$ s'applique non seulement en $x= \pm a$ mais encore en $x= \pm c . \Phi_{2}$ vaut alors :

$$
\frac{p}{2 a \gamma}\left[\sum_{n=0}^{\infty} D_{n} \exp \left(\frac{-n \pi y}{a-c}\right) \cos n \pi\left(\frac{x-c}{a-c}\right)\right] \text {. }
$$

Exprimons ensuite que le potentiel et sa dérivée partielle par rapport à $y$ sont continus en $y=0$

$$
\begin{aligned}
& \sum_{n=0}^{\infty} C_{n} \cos \left(\frac{n \pi x}{a}\right) \quad 0 \leqslant x<c \\
& A_{0}+\frac{1}{2}+\sum_{n=1}^{\infty}\left[A_{n}+\exp \left(\frac{-n \pi y_{0}}{a}\right)\right] \cos \left(\frac{n \pi x}{c}\right)= \\
& \sum_{n=0}^{\infty} D_{n} \cos \left(\frac{n \pi x-c}{a-c}\right) \quad c<x \leqslant a
\end{aligned}
$$

et

$$
\begin{array}{r}
\frac{-1}{c} \sum_{n=1}^{\infty} n C_{n} \cos \left(\frac{n \pi x}{c}\right) 0 \leqslant x<c \\
\frac{1}{a} \sum_{n=1}^{\infty} n\left[A_{n}-\exp \left(\frac{-n \pi y_{0}}{a}\right)\right] \cos \left(\frac{n \pi x}{a}\right)=
\end{array}
$$$$
\frac{-1}{a-c} \sum_{n=1}^{\infty} n D_{n} \cos \left(\frac{n \pi x-c}{a-c}\right) \quad c<x \leqslant a \text {. }
$$ 
En développant les seconds membres en séries de cosinus de période $a$, on déduit de la première relation que, d'une part : $A_{0}+\frac{1}{2}=\frac{c}{a} C_{0}+\frac{a-c}{a} D_{0}$ et que d'autre part avec $k \geqslant 1$

$$
\begin{aligned}
A_{k}+\exp \left(\frac{-k \pi y_{0}}{a}\right)=\frac{2}{\pi}\left\{\frac{1}{k}\left(C_{0}-D_{0}\right) \sin \left(\frac{k \pi c}{a}\right)+\frac{c}{a} \sum_{n=1}^{\infty} C_{n} \int_{0}^{\pi} \cos (n t) \cos \left(\frac{k c t}{a}\right) \mathrm{d} t+\right. \\
\left.+\frac{a-c}{a} \sum_{n=1}^{\infty} D_{n} \int_{0}^{\pi} \cos (n t) \cos \left(\frac{k \pi c}{a}+\frac{k a-c t}{a}\right) \mathrm{d} t\right\} .
\end{aligned}
$$

La seconde relation conduit de son côté à :

$$
\begin{aligned}
A_{k}-\exp \left(\frac{-k \pi y_{0}}{a}\right)=\frac{-2}{k \pi}\left\{\sum_{n=1}^{\infty} n C_{n} \int_{0}^{\pi} \cos (n t) \cos \left(\frac{k c t}{a}\right) \mathrm{d} t\right. & + \\
& \left.+\sum_{n=1}^{\infty} n D_{n} \int_{0}^{\pi} \cos (n t) \cos \left(\frac{k c \pi}{a}+\frac{k a-c t}{a}\right) \mathrm{d} t\right\} .
\end{aligned}
$$

Le système des trois dernières équations est laissé invariant si l'on remplace les composantes continues $A_{0}$, $C_{0}$ et $D_{0}$ par $A_{0}+E, C_{0}+E$ et $D_{0}+E$ où $E$ est une constante; le potentiel est donc déterminé à une constante près et l'on peut poser $A_{0}=0$. On peut en outre, éliminer $A_{k}$ en soustrayant les deux dernières équations membre à membre, on obtient ainsi :

$$
\begin{aligned}
\pi \exp \left(\frac{-k \pi y_{0}}{a}\right)=\sin \left(\frac{k \pi c}{a}\right) \frac{\left(C_{0}-D_{0}\right)}{k}+\sum_{n=1}^{\infty} & \left(\frac{c}{a}+\frac{n}{k}\right) C_{n} \int_{0}^{\pi} \cos (n t) \cos \left(\frac{k c t}{a}\right) \mathrm{d} t+ \\
& +\sum_{n=1}^{\infty}\left(\frac{a-c}{a}+\frac{n}{k}\right) D_{n}\left[\cos \left(\frac{k \pi c}{a}\right) \int_{0}^{\pi} \cos (n t) \cos \left(\frac{k a-c t}{a}\right) \mathrm{d} t\right. \\
& \left.-\cdot \sin \left(\frac{k \pi c}{a}\right) \int_{0}^{\pi} \cos (n t) \sin \left(\frac{k a-c t}{a}\right) \mathrm{d} t\right]
\end{aligned}
$$

et finalement :

$$
\frac{\pi \exp \left(\frac{-k \pi y_{0}}{a}\right)}{\sin \left(\frac{k \pi c}{a}\right)}=\frac{C_{0}-D_{0}}{k}-\frac{c}{a} \sum_{n=1}^{\infty}(-1)^{n} \frac{C_{n}}{n-k \frac{c}{a}}+\frac{a-c}{a} \sum_{n=1}^{\infty} \frac{D_{n}}{n-k \frac{a-c}{a}} .
$$

\section{Bibliographie}

[1] Lambin, Ph. et Troquet, J., J. Appl. Phys. 54 (1983) 4174.

[2] Troquet, J., Lambin, Ph. et Lilien, J. L., Bull. Scient. de l'Association des ingénieurs électriciens sortis de l'Institut Montefiore (A.I.M. Liège) 95 (1982) 59.

[3] Nelson, C. V., Gastongay, P. R., Wilkinson, A. F. and VoukYDIS, P. C., in Vectocardiology, Hoffman I., Hamby R. I. and Glassman E. édit. (North Holland Co. Publ. Amsterdam) 1971, p. 85.

[4] Gabor, D. and Nelson, C. V., J. Appl. Phys. 25 (1954) 413.
[5] TACCARDI, B., Circ. Rés. 12 (1963) 341.

[6] Frank, E., Circ. Res. 1 (1953) 380.

[7] Bayley, R. H., Reynolds, E. W. Jr, Kinard, C. L. and Head, J. F., Circ. Res. 2 (1954) 4.

[8] Nelson, C. V. and Gastongay, P., Circ. Res. 7 (1959) 1039.

[9] Bürger, H. C., Am. Heart J. 49 (1955) 581.

[10] Plonsey, R., IEEE Trans. Biomed. Eng. BME 10 (1963) 3.

[11] Troquet, J., Lambin, Ph. and Nelson, C. V., J. Theor. Biol. (1985) à paraître. 